\title{
Dronedarone in patients with atrial fibrillation
}

\author{
N.M.S. de Groot, C.J. Kirchhof, I.C. van Gelder, J.G. Meeder, A.H.M.M. Balk, A.A Wilde, \\ M.L Simoons
}

Dronedarone is a recently developed new class III antiarrhythmic drug which possesses electrophysiological properties of all four VaughanWilliams classes. An important difference with amiodarone is that it does not contain an iodine component and therefore lacks the iodine-related adverse effects. Based on currently available data, dronedarone can not be recommended as first-line therapy for either rhythm or rate control. We recommend to initiate rhythm or rate control with drugs as indicated in the 2006 guidelines of the ESC and other organisations. As amiodarone, dronedarone can be given to patients for whom standard drug therapy is not effective, or limited by (severe) side effects, although it is less effective than amiodarone. Nevertheless, it may be considered to give dronedarone initially to patients who would otherwise have received amiodarone, since the latter has more severe side effects than the former drug. The daily dosage of dronedarone is oral administration, $400 \mathrm{mg}$ twice daily. Drone-

\author{
N.M.S. de Groot \\ A.H.M.M. Balk \\ M.L Simoons \\ Department of Cardiology, Erasmus Medical Center Rotterdam, \\ the Netherlands \\ C.J. Kirchhof \\ Department of Cardiology, Rijnland Hospital, Leiderdorp, the \\ Netherlands \\ I.C. van Gelder \\ Department of Cardiology, University Medical Center \\ Groningen, Groningen, the Netherlands \\ J.G. Meeder \\ Department of Cardiology, VieCuri Medical Center, Noord- \\ Limburg, Venlo, the Netherlands \\ A.A Wilde \\ Department of Cardiology, Academic Medical Center, \\ Amsterdam, the Netherlands \\ Correspondence to: N.M.S. de Groot \\ Department of Cardiology, Erasmus MC, Thoraxcenter, PO Box \\ 2040, 3000 CA Rotterdam \\ the Netherlands \\ E-mail: nmsdegroot@yahoo.com
}

darone is contraindicated in patients with impaired left ventricular function (NYHA class III/IV) and haemodynamic instability. (Neth Heart J 2010;18:370-3.)

Sleutelwoord: Donedarone; Amiodarone; Anti-Arrhythmia Agents; Atrial Fibrillation

$\mathbf{P}$ harmacological therapy for rate or rhythm control in patients with atrial fibrillation (AF) is often hampered by limited efficacy and (extra)cardiac side effects. ${ }^{1}$ Amiodarone is the most effective antiarrhythmic drug for maintaining sinus rhythm in patients with AF. However, treatment with amiodarone may result in severe side effects ${ }^{2-8}$ (thyroid, pulmonary, neurological and ocular toxicity) which are related to its high iodine content. Dronedarone is a recently developed new class III antiarrhythmic drug $^{9}$ which possesses electrophysiological properties of all four Vaughan-Williams classes. After oral administration steady state plasma levels are reached within four to eight days and the terminal half-life approximates 30 hours. ${ }^{10}$

Similar to amiodarone, dronedarone is a multichannel blocking agent, affecting the rapid activating delayed-rectifier potassium current, the slowly activating delayed-rectifier potassium current, the inward rectifier potassium current, the acetylcholine activated potassium current, peak sodium current and the L-type calcium current. In addition, it exerts antiadrenergic effects. In vitro studies comparing amiodarone and dronedarone have shown that dronedarone is a more potent blocker of peak sodium current, acetylcholine activated potassium current and that it also has stronger antiadrenergic effects. ${ }^{10}$ An important difference with amiodarone is that it does not contain an iodine component and therefore lacks the iodine-related adverse effects.

\section{Rhythm control}

The DAFNE study (Dronedarone Atrial Fibrillation Study after Electrical Cardioversion) showed dose-dependent conversion from AF to sinus 
rhythm after five to seven days of treatment with dronedarone. ${ }^{11}$ The short-term conversion rate varied between 6 and 15\%, at doses between 800 and $1600 \mathrm{mg}$ daily. These conversion rates are similar to those found for amiodarone and sotalol in persistent AF (SAFE-T study). ${ }^{8}$

DAFNE also showed a reverse dose effect on long-term rhythm control; a dose of $400 \mathrm{mg}$ twice daily was associated with the longest median time to first recurrence of $\mathrm{AF}^{11}$

Efficacy of $400 \mathrm{mg}$ dronedarone twice daily compared with placebo for prevention of AF during a follow-up of one year was examined in the EURIDIS (The European trial in Atrial Fibrillation or Flutter Patients receiving dronedarone for the maintenance of sinus rhythm) and ADONIS (the American-Australian-African trial with dronedarone in atrial fibrillation or flutter patients for the maintenance of sinus rhythm) trials. ${ }^{12}$ In both studies dronedarone significantly reduced risk for recurrent AF episodes.

The DIONYSOS trial (Efficacy and safety of dronedarone versus amiodarone for the maintenance of sinus rhythm in patients with $\mathrm{AF}$ ) compared the efficacy and safety of a daily dose of $800 \mathrm{mg}$ dronedarone and $200 \mathrm{mg}$ amiodarone for maintenance of sinus rhythm for at least six months. ${ }^{10}$ At a mean follow-up period of seven months, amiodarone was more effective in maintaining sinus rhythm $(44.7 \%)$ than dronedarone (26.1\%). A recent meta-analysis including four placebo-controlled trials of dronedarone and four placebo-controlled trials of amiodarone also examined the efficacy of both dronedarone and amiodarone for prevention of AF recurrences. Compared with amiodarone, dronedarone appeared to be less effective for prevention of AF recurrences but treatment was associated with fewer side effects. ${ }^{13}$

\section{Rate control}

Rate control during permanent AF by dronedarone was evaluated in the ERATO trial (Efficacy of dronedarone for the prevention of cardiovascular hospitalisation or death from any cause in patients with permanent AF). ${ }^{14}$ The effect of either addition of $800 \mathrm{mg}$ dronedarone daily or placebo to standard rate-control therapy (digitalis, calcium antagonists, $\beta$-blockers) was investigated in patients with permanent AF. Dronedarone reduced the ventricular rate by 11.7 beats $/ \mathrm{min}$. This effect was even more pronounced during exercise (mean reduction of 24.5 beats $/ \mathrm{min}$ ). The ventricular rate control effect with dronedarone was also demonstrated in patients included in the DAFNE, EURIDIS and ADONIS trials. ${ }^{11,12}$ In case of recurrent AF, ventricular rate in patients treated with dronedarone was significantly lower compared with patients treated with placebo.
Effects on cardiovascular morbidity and mortality The ATHENA study ${ }^{15}$ examined the long-term effects of dronedarone treatment $(400 \mathrm{mg}$ twice daily) versus placebo on all-cause mortality and cardiovascular hospitalisations in patients with recurrent episodes of AF. Subjects included in this study were patients with a history of atrial fibrillation/ flutter who were at least 70 years old without additional risk factors, or less than 70 years old with one of the following: hypertension, diabetes, prior cerebrovascular accident, left atrium diameter 50 $\mathrm{mm}$ by M-mode echocardiography or left ventricular ejection fraction $<0.40$ by echocardiography. In this study dronedarone significantly reduced the combined risk of cardiovascular hospitalisation and all-cause mortality. This decrease in cardiovascular hospitalisations in the dronedarone group was a result of fewer admissions for $\mathrm{AF}$, stroke ${ }^{16}$ and acute coronary syndromes. The reduced mortality in the dronedarone group was the result of a reduction in the risk of death from cardiac arrhythmias. ${ }^{15}$

The most frequently observed adverse events of dronedarone were gastrointestinal adverse effects such as nausea and diarrhoea. These were more often observed at higher doses and required drug discontinuation in some patients. ${ }^{10,11,15}$ Thyroid, ocular or pulmonary side effects in these studies were not significantly different from placebo-treated patients. Similar to amiodarone, dronedarone is associated with an increase in serum creatinine. Elevated creatinine levels are assumed to be the result of inhibition of tubular secretion, independent of renal function. ${ }^{17}$ This is particularly the case in patients who use other drugs increasing serum creatinine. ${ }^{12,14}$

The effect of dronedarone on the risk of hospitalisation for progressive heart failure was examined in another placebo-controlled study (ANDROMEDA, heart failure trial with dronedarone in moderate-to-severe congestive heart failure evaluating morbidity decrease). ${ }^{18}$ Patients with New York Heart Association class III or IV congestive heart failure and a left ventricular ejection fraction below $35 \%$ were included and treated with $800 \mathrm{mg}$ dronedarone or placebo. After two months this trial was terminated because of a higher mortality rate in the dronedarone treatment group due to progressive heart failure. No significant differences between placebo and dronedarone patients were seen for arrhythmic events and sudden death. The hypothesis of a depressant effect on left ventricular function cannot be ruled out but is not supported by previous mechanistic studies. A subanalysis revealed that some patients were withdrawn from angiotensinconverting enzyme inhibitors, and/or angiotensin receptor blockers due to an increase in serum creatinine level caused by dronedarone. However, it is uncertain whether this explains the observed excess mortality with dronedarone in these patients. 
Preliminary data showed that dronedarone (doses up to $2000 \mathrm{mg}$ daily) does not affect defibrillation and pacing thresholds while there is a tendency towards reduction of appropriate implantable cardiac defibrillators shocks. ${ }^{10}$

\section{Prolongation of the QT interval}

Experimental models show conflicting evidence regarding the effect of dronedarone on the QT-interval. In anaesthetised dogs with complete chronic AV block, acute administration of dronedarone reduced and homogenised repolarisation whereas chronic administration for four weeks increased the QTc interval. ${ }^{19,20}$ However, this effect was not observed in dogs with normal atrioventricular conduction. ${ }^{20}$ In humans, there was a dose-dependent prolongation of the QT interval, yet torsade de pointes was observed in only one patient. ${ }^{11,12,14} \mathrm{In}$ the DIONYSIS study, ${ }^{10}$ QT prolongation was less frequently observed in subjects using dronedarone compared with amiodarone. Nevertheless, QTc monitoring after initiation of dronedarone therapy is recommended, and interactions with other QTc prolonging drugs or conditions should be avoided until more clinical data are available.

\section{Treatment recommendations}

The studies discussed above indicate that dronedarone is effective for both rhythm and rate control in patients with AF. Dronedarone has shown to be more effective than placebo in maintaining sinus rhythm in patients with recurrent paroxysmal or persistent atrial fibrillation. However, it is less effective in terms of $\mathrm{AF}$ recurrence than amiodarone, but it is associated with fewer side effects. Dronedarone is effective for ventricular rate control in patients with permanent atrial fibrillation in addition to standard antiarrhythmic drugs (digitalis, calcium antagonists, $\beta$-blockers). As compared with placebo, dronedarone significantly reduced ventricular rate. Since dronedarone has been tested in patients also receiving other antiarrhythmic drugs, and no direct comparisons are available between dronedarone and $\beta$-blockers, calcium antagonists or other antiarrhythmic drugs as monotherapy, its relative efficacy compared with such established therapy is not known. Therefore, dronedarone can not be recommended as first-line therapy for either rhythm or rate control.

We recommend to initiate rhythm or rate control with drugs as indicated in the 2006 guidelines of the ESC and other organisations. Dronedarone can be given for maintenance of sinus rhythm in patients with recurrent paroxysmal or persistent atrial fibrillation (rhythm control) in whom standard drug therapy is not effective, or limited by (severe) side effects. Dronedarone is less effective than amiodarone. Nevertheless, it may be considered to give dronedarone initially to patients who otherwise would have received amiodarone, since the latter has more severe side effects than the former drug.

Dronedarone for rate control in patients with permanent atrial fibrillation is indicated in patients in whom other pharmacological agents are ineffective or associated with adverse effects. Similar to rhythm control, dronedarone may be given instead of amiodarone because of the less severe side effects.

Dronedarone appears to be safe and, through mechanisms related to arrhythmia suppression, may reduce vascular events in patients with $\mathrm{AF}$ including hospitalisation for stroke and other causes. However it is contraindicated in patients with impaired left ventricular function (NYHA class III/ IV) and haemodynamic instability.

The daily dosage of dronedarone associated with highest efficacy and safety is oral administration, $400 \mathrm{mg}$ twice daily. Assessment of serum creatinine level after one week on dronedarone is recommended to establish the new steady creatinine level.

\section{References}

1 Fuster V, Ryden LE, Cannom DS, Crijns HJ, Curtis AB, Ellenbogen KA, et al. ACC/AHA/ESC 2006 guidelines for the management of patients with atrial fibrillation-executive summary: a report of the American College of Cardiology/American Heart Association Task Force on Practice Guidelines and the European Society of Cardiology Committee for Practice Guidelines (Writing Committee to Revise the 2001 Guidelines for the Management of Patients with Atrial Fibrillation). Eur Heart J. 2006;27:1979-2030.

2 Babatin M, Lee SS, Pollak PT. Amiodarone hepatotoxicity. Curr Vasc Pharmacol. 2008;6:228-36.

3 Chan AL, Hsieh HJ, Hsieh YA, Lin SJ. Fatal amiodarone-induced hepatotoxicity: a case report and literature review. Int J Clin Pharmacol Ther. 2008;46:96-101.

4 Ernawati DK, Stafford L, Hughes JD. Amiodarone-induced pulmonary toxicity. Br J Clin Pharmacol. 2008;66:82-7.

5 Li J, Tripathi RC, Tripathi BJ. Drug-induced ocular disorders. Drug Saf. 2008;31:127-41.

6 Lloyd MJ, Fraunfelder FW. Drug-induced optic neuropathies. Drugs Today (Barc). 2007;43:827-36.

7 Piga M, Serra A, Boi F, Tanda ML, Martino E, Mariotti S. Amiodarone-induced thyrotoxicosis. A review. Minerva Endocrinol. 2008;33:213-28.

8 Singh BN, Singh SN, Reda DJ, Tang XC, Lopez B, Harris CL, et al. Amiodarone versus sotalol for atrial fibrillation. N Engl J Med. 2005;352:1861-72.

9 Page RL, Hamad B, Kirkpatrick P. Dronedarone. Nat Rev Drug Discov. 2009;8:769-70.

10 Patel C, Yan GX, Kowey PR. Dronedarone. Circulation. 2009;120:636-44.

11 Touboul P, Brugada J, Capucci A, Crijns HJ, Edvardsson N, Hohnloser SH. Dronedarone for prevention of atrial fibrillation: a dose-ranging study. Eur Heart J. 2003;24:1481-7.

12 Singh BN, Connolly SJ, Crijns HJ, Roy D, Kowey PR, Capucci A, et al. Dronedarone for maintenance of sinus rhythm in atrial fibrillation or flutter. N Engl J Med. 2007;357:987-99.

13 Piccini JP, Hasselblad V, Peterson ED, Washam JB, Califf RM, Kong DF. Comparative efficacy of dronedarone and amiodarone for the maintenance of sinus rhythm in patients with atrial fibrillation. J Am Coll Cardiol. 2009;54:1089-95.

14 Davy JM, Herold M, Hoglund C, Timmermans A, Alings A, Radzik D, et al. Dronedarone for the control of ventricular rate in permanent atrial fibrillation: the Efficacy and safety of dRonedArone for the cOntrol of ventricular rate during atrial fibrillation (ERATO) study. Am Heart J. 2008;156:527-9. 
15 Hohnloser SH, Crijns HJ, van Eickels EM, Gaudin C, Page RL, Torp-Pedersen C, et al. Effect of dronedarone on cardiovascular events in atrial fibrillation. N Engl J Med. 2009;360:668-78.

16 Connolly SJ, Crijns HJ, Torp-Pedersen C, van Eickels EM, Gaudin C, Page RL, et al. Analysis of stroke in ATHENA: a placebo-controlled, double-blind, parallel-arm trial to assess the efficacy of dronedarone $400 \mathrm{mg}$ BID for the prevention of cardiovascular hospitalization or death from any cause in patients with atrial fibrillation/atrial flutter. Circulation. 2009;120:1174-80

17 Tschuppert Y, Buclin T, Rothuizen LE, Decosterd LA, Galleyrand J, Gaud C, et al. Effect of dronedarone on renal function in healthy subjects. Br J Clin Pharmacol. 2007;64:785-91.
18 Kober L, Torp-Pedersen C, McMurray JJ, Gotzsche O, Levy S, Crijns $\mathrm{H}$, et al. Increased mortality after dronedarone therapy for severe heart failure. N Engl J Med. 2008; 358:2678-87.

19 van Opstal JM, Schoenmakers M, Verduyn SC, de Groot SH, Leunissen JD, van Der Hulst FF, et al. Chronic amiodarone evokes no torsade de pointes arrhythmias despite QT lengthening in an animal model of acquired long-QT syndrome. Circulation. 2001;104:2722-7.

20 Varro A, Takacs J, Nemeth M, Hala O, Virag L, Iost N, et al. Electrophysiological effects of dronedarone (SR 33589), a noniodinated amiodarone derivative in the canine heart: comparison with amiodarone. Br J Pharmacol. 2001;133:625-34. 